\title{
Disponibilidad léxica en procesos de formación inicial de futuros profesores de inglés
}

\section{Disponibilidade lexical em processos de formação inicial de futuros professores de inglês}

Angie Quintanilla Espinoza*

*Universidad San Sebastián, Concepción / Chile

angie.quintanilla@uss.cl

https://orcid.org/0000-0002-1027-0579

Pedro Salcedo Lagos**

**Universidad de Concepción, Concepción / Chile

psalcedo@udec.cl

https://orcid.org/0000-0002-1741-714X

RESUMEN: A pesar de la riqueza de estudios existentes en el área de disponibilidad léxica, el léxico de las comunidades científicas y/o profesionales ha pasado casi inadvertido por los investigadores. En este contexto, se presentan los resultados de un estudio de disponibilidad léxica en el cual se encuestó a 28 estudiantes de $4^{\circ}$ año de Pedagogía en Inglés en 16 centros de interés (ocho generales y ocho especializados del tipo pedagógico-disciplinar). El objetivo de esta investigación es establecer si existen diferencias cuantitativas entre el léxico general y el léxico especializado de los sujetos de la muestra. Los resultados evidencian que el léxico disponible en los centros de interés de tipo general es mayor que el léxico disponible en los centros especializados. Esto podría deberse a que el léxico de tipo general es de uso cotidiano, mientras que el léxico especializado sólo se utiliza en un ámbito restringido y al interior de una comunidad lingüística específica.

PALABRAS-CLAVE: disponibilidad léxica; léxico especializado; inglés con fines académicos; instrucción basada en contenidos.

RESUMO: Apesar da riqueza de estudos existentes na área de disponibilidade lexical, o léxico das comunidades científicas e/ou profissionais passou quase despercebido pelos pesquisadores. Neste contexto, são apresentados os resultados de um estudo de disponibilidade lexical em que contou com a participação de 28 alunos do $4^{\circ}$ ano de Pedagogia em inglês de 16 centros de interesse (8 gerais e 8 especializados do tipo pedagógico-disciplinar). O objetivo 
desta investigação é saber se existem diferenças quantitativas entre o léxico geral e o léxico especializado dos sujeitos da amostra. Os resultados mostram que o léxico disponível em centros de interesse geral é maior do que o léxico disponível em centros especializados. Isso pode ser devido ao fato de que o léxico de tipo geral é de uso diário, enquanto o léxico especializado é usado apenas em uma área restrita e dentro de uma comunidade linguística específica.

PALAVRAS-CHAVE: disponibilidade lexical; léxico especializado; inglês para fins acadêmicos; instrução baseada em conteúdos.

\section{Introducción}

La adquisición de léxico es un elemento preponderante en el proceso de enseñanza-aprendizaje de una lengua extranjera (LE). De acuerdo a Lightbown y Spada,

nos podemos comunicar utilizando palabras que no están ubicadas en un orden apropiado, pronunciadas perfectamente, o marcadas con el morfema gramatical que corresponde, pero la comunicación frecuentemente se interrumpe si no utilizamos la palabra correcta. (2006, p. 60 , traducción nuestra)

En este mismo escenario, Palapanidi (2012) sugiere que si un estudiante de LE no logra aprender el léxico de la lengua meta es imposible que pueda comunicarse, dado que las palabras portan el significado y este significado es clave en los procesos de comunicación y de enseñanzaaprendizaje.

En este contexto, es necesario señalar que los procesos de formación inicial docente de futuros profesores de inglés se sustentan en la denominada Instrucción Basada en Contenidos, en que el estudiante adquiere de modo paralelo el conocimiento de disciplinas no lingüísticas y las habilidades comunicativas de la lengua meta. De este modo, como señala Stoller (2007, p. 59, traducción nuestra), "el aprendizaje del contenido ayuda al aprendizaje de la lengua, mientras que el dominio de la lengua facilita al estudiante el acceso al contenido en su formación profesional o en su área ocupacional". Es así que el léxico de los estudiantes de una lengua extranjera cobra relevancia, puesto que es este conocimiento que les permitirá comunicarse y comprender de forma adecuada las temáticas que forman parte de su proceso de formación docente tanto a nivel de competencia lingüística como pedagógica. 
La presente investigación tiene como propósito realizar un análisis de la disponibilidad léxica de estudiantes de último año de la carrera de Pedagogía en Inglés con el fin de establecer si existen diferencias entre los centros de interés de tipo general ${ }^{1}$ y especializado ${ }^{2}$. Este interés surge debido a que en la actualidad no existen estudios de disponibilidad léxica en inglés a nivel universitario que permitan conocer y establecer diferencias, si es que existen, entre el léxico disponible general y el especializado. A fin de poder conocer este léxico y establecer posibles diferencias, se procederá a cuantificar el léxico disponible utilizando los estadígrafos que determinan la riqueza léxica, se compararán estos índices y finalmente se describirá el léxico disponible entregado por los sujetos que forman parte de la muestra.

El presente artículo está organizado en las siguientes secciones: en la sección 2 se desarrollan los fundamentos teóricos de la disponibilidad léxica, se presentan estudios en inglés y en léxico especializado. En la sección 3 se explicita el diseño de la investigación. En la sección 4 se examinan los resultados obtenidos. Finalmente, en la sección 5 se presentan las conclusiones del estudio.

\section{Fundamentos teóricos}

\subsection{Disponibilidad léxica}

Los estudios de disponibilidad léxica se originan en Francia en los años cincuenta de la mano de un grupo de investigadores franceses que trabajaban en la elaboración del Français élémentaire (GOUGENHEIM et al., 1956). El objetivo de estos investigadores era elaborar una "lengua base" a partir de una gramática y un léxico esencial para enseñar francés a inmigrantes y a habitantes de excolonias francesas.

Al principio su trabajo se centró en la elaboración de léxico frecuente, a partir de la premisa de que las palabras que se utilizan con mayor frecuencia deben ser enseñadas primero. Sin embargo, pronto quedó de manifiesto que este criterio de selección no era el más apropiado dado que en los listados

\footnotetext{
${ }^{1}$ Se define como léxico general el vocabulario relacionado con temáticas de dominio general (partes del cuerpo, partes de la casa, miembros de la familia etc.).

${ }^{2}$ Se define como léxico especializado el léxico relacionado con la disciplina o área de especialización (Pedagogía en inglés).
} 
de frecuencia las palabras esenciales para la comunicación, no aparecían o aparecían en posiciones muy retrasadas en la lista. Estos estudios de frecuencia léxica dieron como resultado una lista de frecuencias decrecientes que incluyeron: $1^{\circ}$ palabras gramaticales, $2^{\circ}$ verbos, $3^{\circ}$ adjetivos y $4^{\circ}$ algunos sustantivos de carácter general.

A partir de estos resultados, los investigadores decidieron cambiar el foco de su trabajo y centrarse en la presencia de las palabras en el discurso, y establecieron diferencias entre léxico frecuente y léxico disponible. El léxico frecuente incluye palabras que aparecen siempre, independientemente de la materia de que se trate (palabras atemáticas), mientras que el léxico disponible incluye palabras que solo aparecen cuando se trata de determinada materia (palabras temáticas). Al respecto, Gougenheim (1967) señala que las palabras disponibles son aquellas que forman parte del lexicón mental de un hablante y que son utilizadas sólo cuando las circunstancias lo requieren. Del mismo modo, Michéa (1953, p. 4, traducción nuestra) afirma que

una palabra disponible es una palabra que, sin ser particularmente frecuente, siempre está lista para ser usada y se presenta de forma inmediata y natural a la mente en el momento en que se la necesita. Es una palabra que formando parte de asociaciones de ideas usuales, existe en potencia en el hablante, en cuanto entran en juego dichas asociaciones.

De igual manera, autores contemporáneos como Hernández y Tomé (2017, p. 100) definen el léxico disponible como "un conjunto de unidades léxicas que están altamente disponibles para un hablante de una lengua concreta cuando se le pregunta por una determinada categoría semántica".

Este léxico disponible se obtiene a partir de pruebas asociativas a centros de interés, en que se les pide a los hablantes que enuncien o escriban, en un período de dos minutos de tiempo (MENA, 1986), las palabras que asocian con una determinada temática o centro de interés. Garzón y Penagos (2016) justifican esta limitación en el tiempo de respuesta como una forma de obtener un léxico más natural y espontáneo (más disponible), y a la vez evitar la aparición de un léxico rebuscado, artificioso y poco natural.

\subsection{Disponibilidad léxica en inglés}

A pesar de la importancia del inglés en la actualidad, los estudios en el área de disponibilidad léxica en esta lengua son aún escasos. $\mathrm{Al}$ respecto, Šifrar Kalan (2015) señala que esto se debe a que a la investigación en torno 
al vocabulario ha seguido distintos caminos en la Lingüística Aplicada Hispánica y en la Angloparlante.

Entre los estudios más relevantes en inglés como LE o segunda lengua (L2) destacan investigaciones realizadas en España (FERNÁNDEZ, 2010; JIMÉNEZ et al., 2014; JIMÉNEZ CATALÁN; OJEDA ALBA, 2010; LUGONES, 2015), Eslovenia (ŠIFRAR KALAN, 2015) y Chile (FERREIRA; ECHEVERRÍA, 2010; GERMANY; CARTES, 2000; ZUBANOV, 2006). En general, en estos trabajos se observa un interés en la relación que existe entre variables sociales (género, edad, nivel sociocultural, tipo de establecimiento educacional, años de estudio de la lengua etc.) y afectivas (motivación) en el rendimiento en los test de disponibilidad léxica en inglés como LE. También se han realizado estudios con el propósito de comparar la disponibilidad léxica en inglés con alguna lengua materna como el español y el esloveno (ŠIFRAR KALAN, 2015), y establecer diferencias entre el léxico disponible de hablantes nativos de inglés y estudiantes de inglés de nivel avanzado, incluyendo las diferencias en la organización mental del léxico (FERREIRA; ECHEVERRÍA, 2010).

\subsection{Disponibilidad léxica especializada}

López Morales (1999, p. 11) indica que los vocablos que posee un hablante "no se actualizan a menos que se necesiten para comunicar una información muy específica”. Al respecto, Urzúa, Sáez y Echeverría (2006) plantean la necesidad de estudiar qué ocurre con el léxico disponible de hablantes que se desenvuelven en un contexto especializado y que son parte de una comunidad de habla que utiliza un léxico técnico y/o científico para interactuar de forma efectiva con otros miembros al interior de dicha comunidad.

De este modo, el léxico disponible de estas comunidades es restrictivo desde una perspectiva cuantitativa, porque se limita a un grupo restringido dentro de una comunidad de habla mayor, y desde una perspectiva cualitativa, ya que se vincula a un sector específico por la profesión de los hablantes (NAVARRO MARRERO, 2009).

Entre los estudios que se han centrado en léxico especializado podemos mencionar los realizados por Guerra Salas y Gómez Sánchez (2003) y Gómez Parra y Guerra Salas (2004) en el área de los medios de comunicación, Urzúa, Sáez y Echeverría (2006), Salcedo Lagos y Del Valle (2013), Ferreira, Salcedo Lagos y Del Valle (2014), Salcedo Lagos, Del Valle, 
Quintanilla Espinoza y Zambrano (2016) y Pacheco, Ponce Castañeda y Palomares Sánchez (2016) en el área de las matemáticas, Medina (2009) en el área jurídica, Navarro Marrero (2009) en el área de la fisioterapia y Quintanilla Espinoza y Salcedo Lagos (2019) en el área pedagógica. En general, en estas investigaciones se observa un especial interés en estudiar la evolución del léxico especializado a medida que aumentan los años de estudios de los sujetos. Además, en algunos casos se contrasta el léxico de los estudiantes con expertos, ya sea profesores o profesionales en ejercicio. Los resultados de estos estudios evidencian un crecimiento del léxico disponible de los alumnos a medida que éstos aumentan sus años de estudio (SALCEDO LAGOS; DEL VALLE, 2013; URZÚA; SÁEZ; ECHEVERRÍA, 2006), asimismo, se observa que los sujetos presentan un léxico más técnico o especializado (GÓMEZ PARRA; GUERRA SALAS, 2004) y que las respuestas se hacen más pertinentes al compararlas con las entregadas por los especialistas (MEDINA, 2009).

En el área de disponibilidad léxica especializada, también se han desarrollado algunos estudios tendientes a conocer el léxico de futuros docentes en el área de enseñanza de español como lengua extranjera (PEDRONI TORRES, 2015) y en educación infantil y primaria (HERRANZ LLÁCER, 2018). El trabajo de Pedroni Torres (2015) se centra en una comunidad lingüística especializada que presenta un aumento en los vocablos producidos en los niveles más avanzados, sin embargo, los centros de interés en estudio son de tipo general. Por otro lado, Herranz Llácer (2018) incorpora los centros especializados TICs y Educación. Entre sus hallazgos se observa un cambio en la perspectiva de los estudiantes a medida que avanzan en sus estudios y tienen una mayor exposición a prácticas pedagógicas, dado que en el corpus se emplean más palabras desde la perspectiva del profesor. No obstante, esta evolución en el léxico no es tan evidente e implicaría la necesidad de un reforzamiento en las distintas áreas del conocimiento presentes en el currículum.

Cabe señalar que la investigación en el área de disponibilidad léxica en léxico específico o especializado es aún incipiente, y que la aplicabilidad de este tipo de investigación puede tener implicancias pedagógicas en diversas áreas del saber, incluyendo el área de la enseñanza-aprendizaje de segundas lenguas, que es la base de esta investigación. Un ejemplo de esta aplicabilidad es la posibilidad de utilizar un corpus de léxico disponible específico en la creación de diccionarios especializados. 


\section{Diseño de la investigación}

\subsection{Problema}

En lo referido al aprendizaje de lenguas, y particularmente en procesos de formación de profesores de LE, el léxico cumple un rol fundamental dado que sostiene los procesos de aprendizaje (BONORINO; CUÑARRO, 2006; VALENCIA et al., 1992) y permite que el futuro profesor de inglés pueda cumplir con los requerimientos lingüísticos que plantea el Marco Común Europeo de Referencia (MCER) para las lenguas a nivel C1 y, a su vez, acceder a los conocimientos propios de la disciplina a partir de la adquisición de léxico especializado en el área.

De este modo, cabe preguntarse si es posible cuantificar y describir el léxico de estudiantes de Pedagogía en Inglés en centros de interés generales y específicos. Para esto, se utilizará el modelo de disponibilidad léxica, considerando tres índices: promedio de respuestas (XR), total de palabras diferentes (NPD), e índice de cohesión (IC). De acuerdo a Cepeda, Granada y Pomes (2014), estos índices son los que más entregan mayor información con respecto a la riqueza léxica de los sujetos en estudio.

\subsection{Objetivos}

La presente investigación tiene como objetivo conocer el léxico disponible de tipo general y específico que posee una muestra de estudiantes de Pedagogía en Inglés.

\subsection{Hipótesis de la investigación}

El promedio del léxico disponible de los estudiantes en los centros de interés de tipo general es mayor que el especializado.

\subsection{Muestra}

La muestra para esta investigación está compuesta por 28 estudiantes universitarios -quienes participaron de forma voluntaria- dentro de un universo de 35 alumnos. Los participantes son hablantes de español como lengua materna, que cursan $4^{\circ}$ año de Pedagogía en Inglés y se encuentran en la etapa final de su proceso de formación. Su nivel de competencia lingüística en LE corresponde al B2-C1. 


\subsection{Instrumento (centros de interés)}

Los centros de interés investigados se obtuvieron a partir de la revisión de los contenidos léxicos incluidos en los programas del Ministerio de Educación de Chile (Mineduc) para el subsector de inglés desde el $1^{\circ}$ básico al $4^{\circ}$ medio (generales) y de los estándares disciplinarios de inglés para la formación de profesores establecidos por el Mineduc en el 2014 especializados- (CHILE, 2014). La pertinencia de estos centros de interés fue evaluada por nueve profesores universitarios de inglés como lengua extranjera. A partir del análisis de las respuestas emitidas por los evaluadores, se obtuvieron ocho centros de interés de tipo general y ocho centros de tipo especializado. De este modo, el test de disponibilidad léxica queda conformado por los campos léxicos presentes en la Tabla 1.

En resumen, el test de disponibilidad léxica incluyó dos apartados (ver Anexo A), el primero recopila información de identificación del sujeto (edad y género); mientras que el segundo incorpora los 16 centros de interés en estudio, para los cuales los sujetos deben producir una lista de palabras en un tiempo de dos minutos por centro, en concordancia con las investigaciones actuales en la materia.

Las instrucciones incluidas en el test de disponibilidad léxica son leídas en voz alta por el encuestador a todos los sujetos que forman parte de la muestra. Una vez que comienza la evaluación se presentan cada uno de los centros de interés de forma individual y se cronometran los dos minutos correspondientes. El test tuvo una duración de 32 minutos. 
TABLA 1 - Centros de interés para el test de disponibilidad léxica

\begin{tabular}{l|l}
\hline \multicolumn{1}{c|}{ Generales } & \multicolumn{1}{c}{ Especializados } \\
\hline $\begin{array}{l}\text { Family members } \\
\text { Miembros de la familia }\end{array}$ & $\begin{array}{l}\text { Language components } \\
\text { Componentes lingüísticos }\end{array}$ \\
\hline $\begin{array}{l}\text { Food and drink } \\
\text { Comida y bebida }\end{array}$ & $\begin{array}{l}\text { Lesson planning } \\
\text { Planificación de clases }\end{array}$ \\
\hline $\begin{array}{l}\text { Occupations } \\
\text { Profesiones y oficios }\end{array}$ & $\begin{array}{l}\text { Methods and approaches in language teaching } \\
\text { Enfoques y métodos en la enseñanza de lenguas }\end{array}$ \\
\hline $\begin{array}{l}\text { Hobbies and free time activities } \\
\text { Pasatiempos y actividades de tiempo libre }\end{array}$ & $\begin{array}{l}\text { Integrated language skills } \\
\text { Habilidades lingüísticas integradas }\end{array}$ \\
\hline $\begin{array}{l}\text { Feelings and emotions } \\
\text { Sentimientos y emociones }\end{array}$ & $\begin{array}{l}\text { Assessment } \\
\text { Evaluación }\end{array}$ \\
\hline $\begin{array}{l}\text { Body parts } \\
\text { Partes de cuerpo }\end{array}$ & $\begin{array}{l}\text { Receptive skills } \\
\text { Habilidades receptivas }\end{array}$ \\
\hline Colors & $\begin{array}{l}\text { Productive skills } \\
\text { Colores }\end{array}$ \\
\hline $\begin{array}{l}\text { The weather } \\
\text { El clima }\end{array}$ & $\begin{array}{l}\text { Reflectivity / Reflection } \\
\text { Reflectividad/Reflexión }\end{array}$ \\
\hline
\end{tabular}

\subsection{Procesamiento de los datos}

Una vez recopilados los datos se procedió a validar las respuestas y limpiar el corpus (lematizado). Para esto se consideraron aspectos como la ortografía variada para una misma palabra, la anotación en singular de sustantivos y adjetivos, el uso de guiones para unir las palabras compuestas, la remisión de las formas verbales al infinitivo, con la excepción del gerundio. Al finalizar este procedimiento, se sometió el corpus a un procesamiento computacional para el cálculo de índices y frecuencias, usando el software LexMath (2013).

La información obtenida en el test de disponibilidad léxica se analizó considerando los tres estadígrafos que permiten establecer con mayor claridad la riqueza léxica de los sujetos de la muestra; a saber, el promedio de respuestas (XR), el total de palabras diferentes (PD) y el índice de cohesión (IC). A continuación, se describe cada uno de estos índices de acuerdo a Ferreira, Salcedo Lagos y Del Valle (2014): 
- El promedio de respuestas (XR) indica cuántos son los vocablos que, en promedio, poseen los sujetos para su comunicación en el ámbito de conocimiento que explora el centro de interés respectivo;

- El total de palabras diferentes (NPD) es un índice que da cuenta del total de vocablos conocidos por el grupo muestral;

- El índice de cohesión (IC) es un indicador del grado de coincidencia en las respuestas de los sujetos.

Además de estos tres índices, se consideró el índice de disponibilidad léxica (IDL), que determina el grado de disponibilidad de un vocablo en la mente del hablante.

\section{Análisis de los resultados}

En este apartado se presenta un análisis global de los datos obtenidos. La Tabla 2 presenta los resultados generales de la muestra, considerando solo los centros de interés de tipo general. En esta tabla se observa que los centros de interés con mayor número de palabras diferentes (NPD) son feelings and emotions $(\mathrm{NPD}=215)$ y bobbies $(\mathrm{NPD}=182)$. Mientras que el NPD más bajo se evidencia en family members $(\mathrm{NPD}=65)$ y colors $(\mathrm{NPD}=95)$, lo que podría deberse a que estos centros poseen un inventario de vocablos cerrado o limitado (MATEO, 1998).

Con respecto al promedio de palabras (XR) entregadas por los sujetos de la muestra, es posible indicar que los centros de interés con resultados más altos son body parts con un promedio de 25 palabras por sujeto $(\mathrm{XR}=25,39)$ y food and drink $(\mathrm{XR}=20,79)$. El promedio de palabras más bajo se observa en hobbies $(\mathrm{XR}=15,11)$ y occupations $(\mathrm{XR}=15,36)$.

Por último, se evidencia que los índices de cohesión (IC) más altos se observan en family members ( $\mathrm{IC}=0,29835)$ y body parts ( $\mathrm{IC}=0,22081)$. Estos resultados indican que los sujetos de la muestra tienen un mayor grado de coincidencia en respuestas en estos centros de interés, por lo tanto, podemos señalar que estos centros son más densos y cerrados (ECHEVERRÍA et al., 1987). Por otro lado, los centros de interés más abiertos y menos cohesionados presentan un IC más bajo; este es el caso de feelings and emotions (IC=0,07542) y bobbies ( $\mathrm{IC}=0,08301)$. 
TABLA 2 - Resultados generales en centros de interés de tipo general

\begin{tabular}{l|c|c|c|c}
\hline \multicolumn{1}{c|}{ Centros de interés } & $\mathbf{N}^{\circ}$ Palabras & XR & NPD & IC \\
\hline Family members & 543 & 19,39 & 65 & 0,29835 \\
\hline Food and drink & 582 & 20,79 & 152 & 0,13675 \\
\hline Occupations & 430 & 15,36 & 155 & 0,09908 \\
\hline Hobbies & 423 & 15,11 & 182 & 0,08301 \\
\hline Feelings and emotions & 454 & 16,21 & 215 & 0,07542 \\
\hline Body parts & 711 & 25,39 & 115 & 0,22081 \\
\hline Colors & 436 & 15,57 & 95 & 0,16391 \\
\hline Weather & 441 & 15,75 & 135 & 0,11667 \\
\hline
\end{tabular}

La Tabla 3 presenta los resultados generales de la muestra, considerando solo los centros de interés de tipo especializado. En esta tabla se observa que los centros de interés con mayor número de palabras diferentes (NPD) son productive skills $(\mathrm{NPD}=167)$ y lesson planning $(\mathrm{NPD}=162)$. Por otro lado, el valor de NPD más bajo se refleja en los centros methods and approaches $(\mathrm{NPD}=87)$ y language components $(\mathrm{NPD}=124)$.

En relación al promedio de palabras (XR) entregadas por los sujetos es posible señalar que los centros de interés con los mayores resultados son lesson planning $(\mathrm{XR}=15,39)$ y language components $(\mathrm{XR}=12,46)$. Mientras que el promedio de palabras más bajo se observa en reflectivity $(\mathrm{XR}=8,04)$ y methods and approaches $(\mathrm{XR}=8,11)$.

Finalmente, el índice de cohesión (IC) de los centros de interés especializados se presenta como sigue: language components $(\mathrm{IC}=0,10052)$ y lesson planning $(\mathrm{IC}=0,09502)$ presentan los resultados más altos, por el contrario, los centros reflectivity (IC=0,05580) y productive skills (IC=0,06630) son los más abiertos y menos cohesionados dado que presentan el IC más bajo de los centros en estudio. 
TABLA 3 - Resultados generales en centros de interés de tipo especializado

\begin{tabular}{l|c|c|c|c}
\hline \multicolumn{1}{c|}{ Centros de interés } & $\mathbf{N}^{\circ}$ Palabras & XR & NPD & IC \\
\hline Language components & 349 & 12,46 & 124 & 0,10052 \\
\hline Lesson planning & 431 & 15,39 & 162 & 0,09502 \\
\hline Methods and approaches & 227 & 8,11 & 87 & 0,09319 \\
\hline Integrated language skills & 281 & 10,04 & 134 & 0,07489 \\
\hline Assessment & 324 & 11,57 & 152 & 0,07613 \\
\hline Receptive skills & 298 & 10,64 & 156 & 0,06822 \\
\hline Productive skills & 310 & 11,07 & 167 & 0,06630 \\
\hline Reflectivity & 225 & 8,04 & 144 & 0,05580 \\
\hline
\end{tabular}

Luego de realizar un análisis independiente de los centros de interés de tipo general y especializado, se presenta una comparación en cada uno de los índices ya analizados. La Figura 1 muestra el número de palabras diferentes en cada uno de los centros de interés estudiados; como se había mencionado, se observa que los valores más altos se encuentran en los centros generales feelings and emotions $(\mathrm{NPD}=215)$ y bobbies $(\mathrm{NPD}=182)$. Por otro lado, los valores más bajos se observan el centro family members con un $\mathrm{NPD}=65$ (centro de tipo general) y methods and approaches con un NPD $=87$ (centro de tipo específico).

FIGURA 1 - Número de palabras diferentes en los centros de interés

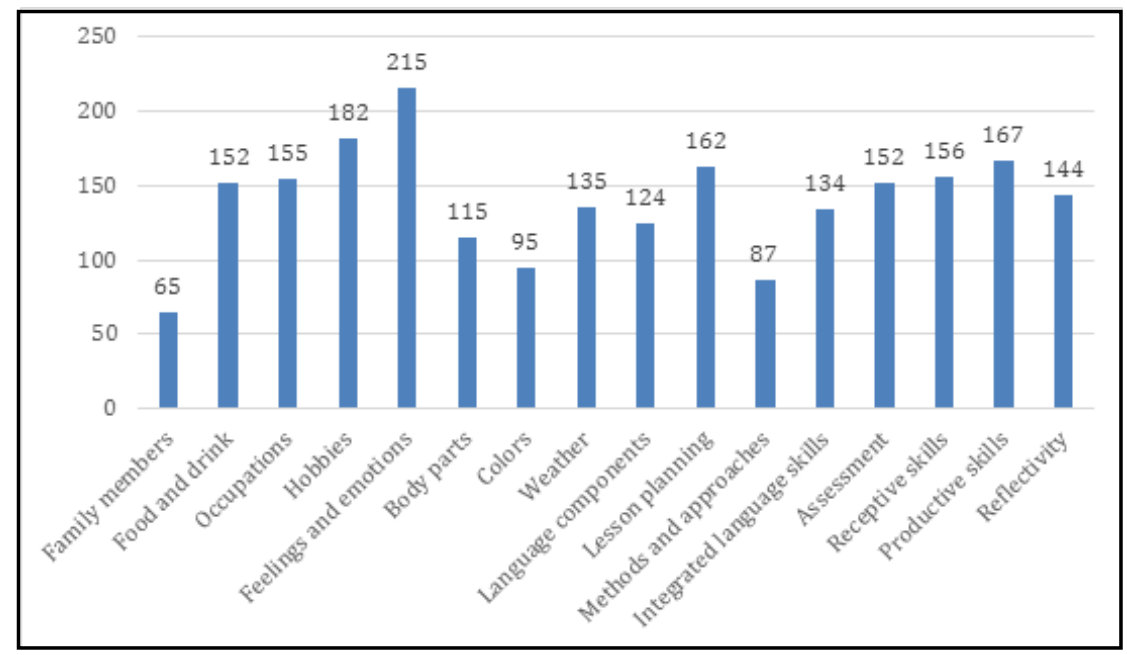


En la Figura 2 es posible ver que el promedio de palabras en los centros de tipo general es, en casi todos los casos, mayor que en los centros de tipo especializado. Esto se observa en hobbies $(\mathrm{XR}=15,11)$ y occupations $(\mathrm{XR}=15,36)$ que poseen un promedio menor al centro lesson planning que posee un $\mathrm{XR}=15,39$.

FIGURA 2 - Promedio de palabras (XR) en los centros de interés

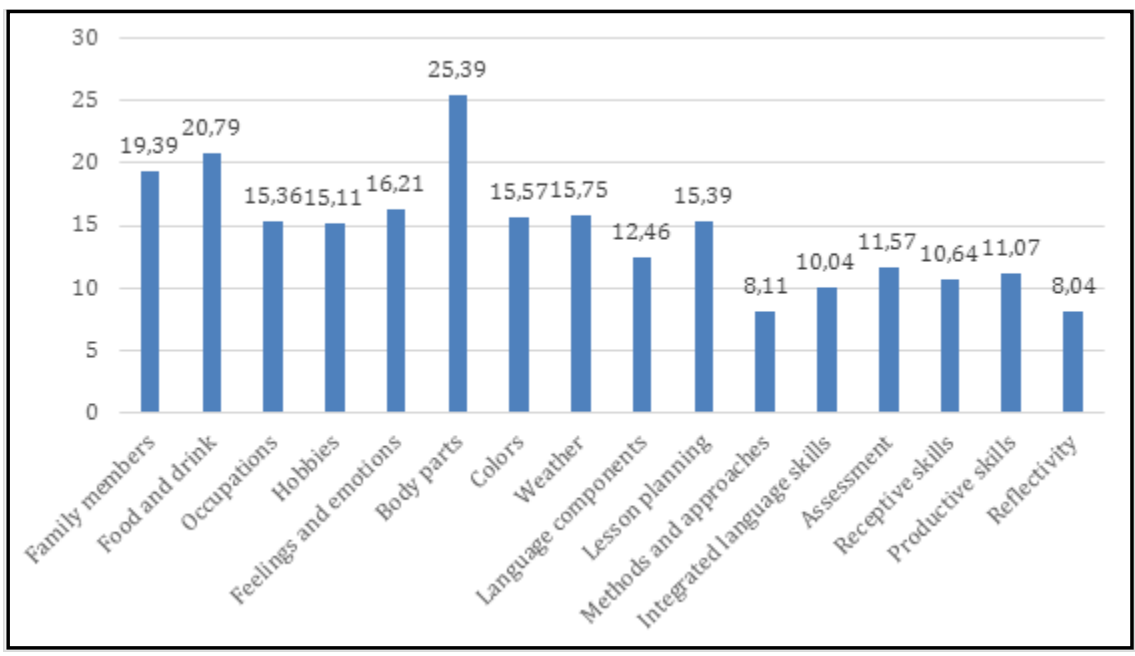

FIGURA 3 - Índice de cohesión (IC) por centro de interés

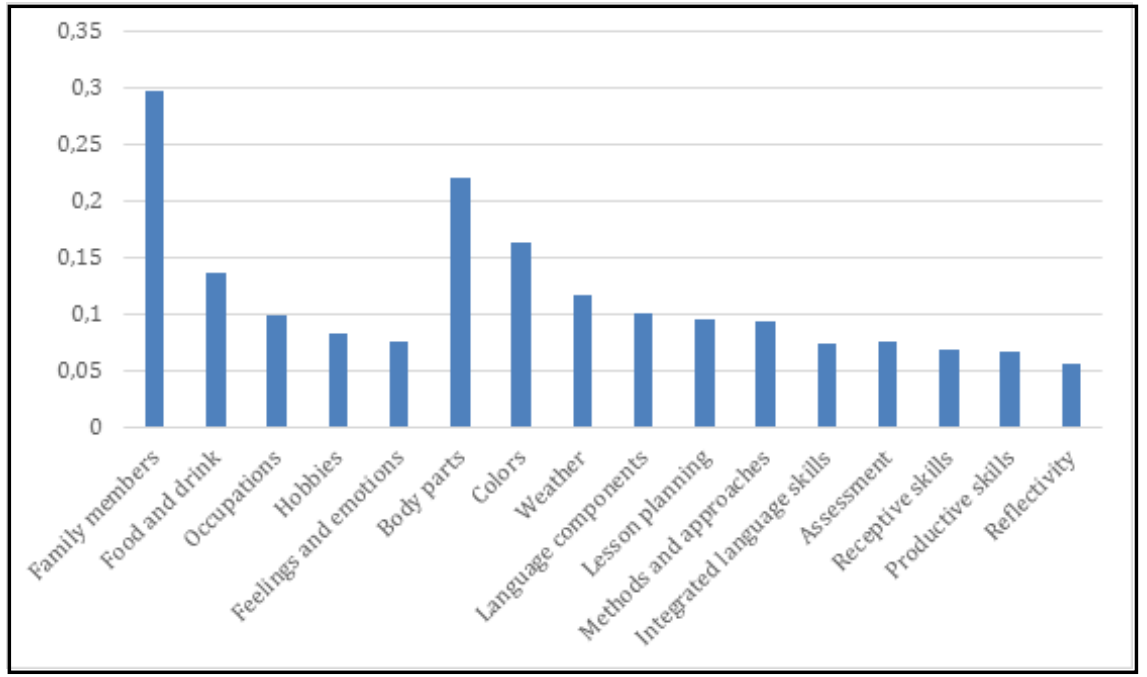


La Figura 3 ilustra el índice de cohesión de los diferentes centros de interés. En este caso, se observa que los centros de tipo general presentan un IC mayor que el de los centros especializados. Sin embargo, destaca el centro language components (IC $=0,10052)$ con un IC mayor que el de los centros hobbies $(0,08301)$ y feelings and emotions $(0,07542)$.

La Tabla 4 presenta una comparación de los resultados generales (promedio) de acuerdo al tipo de centro de interés, que incluye los tres índices utilizados como medida de análisis, además del número de palabras mencionadas por los sujetos de la muestra. Los resultados evidencian que los centros de interés de tipo general superan a los centros de tipo especializado en el número de palabras totales ( general $=502,5-$ especializado $=305,625$ ), del promedio de respuestas (general $=17,94625-$ especializado $=10,915) \mathrm{y}$ del índice de cohesión (general $=0,14925-$ especializado=0,07875875). Sin embargo, no se advierte una diferencia sustancial con respecto al número de palabras diferentes (general=139,25 - especializado=140,75).

Con el propósito de determinar si las diferencias que se observan entre los centros de interés de tipo general y especializado tienen algún grado de significancia estadística, se aplicó la prueba de normalidad Shapiro-Wilk, que arrojó una distribución normal de los datos. A partir de este resultado, se utilizó la prueba T para muestras independientes. Los datos obtenidos evidencian que sólo existen diferencias estadísticamente significativas en el número de palabras totales, en el XR y en el IC $(\mathrm{p}<0.05)$.

Con respecto al NPD, cabe señalar que a pesar de que la cifra que se recoge en el corpus es muy similar en ambos tipos de centros de interés, se constata una diferencia en el IC que implicaría la existencia de un alto nivel de similitud en el léxico general de los sujetos de la muestra, lo que no ocurre en el caso del léxico especializado.

TABLA 4 - Resultados generales de acuerdo al tipo de centro de interés

\begin{tabular}{c|c|c|c|c}
\hline Centros de interés & $\mathbf{N}^{\circ}$ Palabras & XR & NPD & IC \\
\hline Generales & 502,5 & 17,94625 & 139,25 & 0,14925 \\
\hline Especializados & 305,625 & 10,915 & 140,75 & 0,07875875 \\
\hline
\end{tabular}

A continuación, con el objetivo de describir el léxico disponible de los sujetos de la muestra se presentan en orden decreciente de acuerdo al IDL las 10 primeras palabras en los centros de interés estudiados. Primero 
se muestran los centros de tipo general (ver Tabla 5 y 6 ) y luego los especializados (ver Tabla 7, 8 y 9).

En las Tablas 5 y 6 figuran las 10 primeras palabras disponibles en los centros de interés de tipo general. A continuación, se presentan algunas ideas centrales con respecto a los datos que se observan en la tabla 5:

- En el centro de interés family members se observa que las cuatro palabras más disponibles son mother, father, sister y brother. Estas palabras representan el estereotipo de un núcleo familiar;

- En el centro de interés food and drink aparecen dos bebidas alcohólicas (beer y wine) entre los 10 primeros ítems léxicos. Esto podría deberse a que los sujetos de la muestra son estudiantes universitarios y a que el alcohol es parte de sus experiencias de vida adulta;

- En el centro de interés occupations, la palabra con el IDL más alto es teacher. La relevancia de este vocablo podría estar relacionada con la futura profesión de los sujetos de la muestra, quienes se encuentran a un mes de terminar su carrera profesional y convertirse en profesores.

TABLA 5 - Léxico disponible en los centros de interés family members, food and drink, occupations y weather

\begin{tabular}{c|l|c|l|c|c|c|c|c}
\hline \multirow{2}{*}{} & \multicolumn{2}{|c|}{ Family members } & \multicolumn{2}{c|}{ Food and drink } & \multicolumn{2}{c}{ Occupations } & \multicolumn{2}{c}{ Weather } \\
\cline { 2 - 9 } & \multicolumn{1}{c|}{ Palabra } & IDL & Palabra & IDL & Palabra & IDL & Palabra & IDL \\
\hline 1 & Mother & 0,843891 & Juice & 0,401563 & Teacher & 0,705031 & Sunny & 0,728794 \\
\hline 2 & Father & 0,827718 & Apple & 0,304536 & Doctor & 0,566994 & Cloudy & 0,562006 \\
\hline 3 & Sister & 0,604521 & Beer & 0,285517 & Nurse & 0,423746 & Rainy & 0,513751 \\
\hline 4 & Brother & 0,519600 & Soda & 0,277693 & Engineer & 0,318906 & Windy & 0,419349 \\
\hline 5 & Grandmother & 0,488560 & Water & 0,265792 & Singer & 0,284332 & Cold & 0,306937 \\
\hline 6 & Grandfather & 0,472977 & French-fries & 0,243994 & Lawyer & 0,255783 & Rain & 0,247216 \\
\hline 7 & Uncle & 0,379287 & Bread & 0,228872 & Policeman & 0,221320 & Snowy & 0,241831 \\
\hline 8 & Cousin & 0,362018 & Hot-dog & 0,219578 & Actor & 0,201956 & Sun & 0,231160 \\
\hline 9 & Aunt & 0,338731 & Orange & 0,208019 & Secretary & 0,183973 & Hot & 0,228436 \\
\hline 10 & Son & 0,321446 & Wine & 0,205052 & Firefighter & 0,174019 & Warm & 0,215912 \\
\hline
\end{tabular}


A continuación, se presentan algunas ideas generales con respecto a los datos que se observan en la Tabla 6:

- En el centro de interés feelings and emotions se destaca el vocablo blue que en el contexto de este centro corresponde a una expresión idiomática utilizada en expresiones como: I'm blue o I'm feeling blue (estoy triste o me siento triste);

- En el centro de interés colors se observa la presencia de colores primarios (red, blue, yellow), secundarios (green, orange, purple) y de los vocablos que representan la ausencia de color (black) y la presencia de todos los colores (white). Estos vocablos aparecen en la lista en forma consecutiva (posición 3 y 4), lo que indicaría que existe una asociación mental al evocar estos ítems que correspondería a una expresión binomial en inglés (black and white) que difiere de la expresión en español "blanco y negro" (white and black).

TABLA 6 - Léxico disponible en los centros de interés feelings and emotions, body parts, colors y bobbies

\begin{tabular}{l|l|c|l|c|l|c|l|c}
\hline \multirow{2}{*}{} & \multicolumn{2}{|l|}{ Feelings and emotions } & \multicolumn{2}{c|}{ Body parts } & \multicolumn{2}{c|}{ Colors } & \multicolumn{2}{c}{ Hobbies } \\
\cline { 2 - 9 } & Palabra & IDL & Palabra & IDL & Palabra & IDL & Palabra & IDL \\
\hline 1 & Sad & 0,675488 & Head & 0,652951 & Red & 0,817798 & Reading & 0,404875 \\
\hline 2 & Happy & 0,675336 & Eye & 0,566525 & Blue & 0,805681 & $\begin{array}{l}\text { Listening-to- } \\
\text { music }\end{array}$ & 0,304876 \\
\hline 3 & Angry & 0,414364 & Hair & 0,556906 & Black & 0,591577 & Playing-football & 0,224993 \\
\hline 4 & Happiness & 0,258451 & Leg & 0,442123 & White & 0,578377 & Swimming & 0,202540 \\
\hline 5 & Sadness & 0,255888 & Nose & 0,418522 & Green & 0,540987 & Skating & 0,199282 \\
\hline 6 & Excited & 0,201277 & Ear & 0,414260 & Yellow & 0,539069 & Running & 0,185652 \\
\hline 7 & Upset & 0,120613 & Arm & 0,396474 & Orange & 0,501300 & Watching-tv & 0,184323 \\
\hline 8 & Blue & 0,118243 & Mouth & 0,395330 & Pink & 0,466473 & $\begin{array}{l}\text { Playing-video- } \\
\text { games }\end{array}$ & 0,181112 \\
\hline 9 & Tired & 0,108536 & Finger & 0,295701 & Purple & 0,443453 & Skiing & 0,166719 \\
\hline 10 & Enthusiastic & 0,101335 & Shoulder & 0,252537 & Brown & 0,420255 & Singing & 0,158115 \\
\hline
\end{tabular}


En las Tablas 7, 8 y 9 se presentan las 10 primeras palabras disponibles en los centros de interés de tipo especializado. A continuación, se muestran algunas ideas generales con respecto a los datos que se observan en la Tabla 7:

- En el centro de interés language components se observa que las tres palabras con el IDL más alto corresponden a noun, verb y adjective que corresponden a partes de una oración, además, en las primeras 10 palabras aparecen otras partes de la oración tales como pronoun y adverb. También aparecen en este listado los dos elementos fundamentales de la oración que son el subject y predicate;

- En el centro de interés lesson planning destaca el léxico relacionado con los objetivos de la planificación como son subsidiary-aim, main-aim, aim y personal aim;

- En el centro de interés receptive skills se visualizan con el IDL léxico más alto las dos habilidades receptivas (listening y reading), y también se observan sub-habilidades como skimming, scanning, gist y listening for gist.

TABLA 7 - Léxico disponible en los centros de interés language components, lesson planning y receptive skills

\begin{tabular}{l|l|c|l|c|l|c}
\hline & \multicolumn{2}{|l|}{ Language components } & \multicolumn{2}{c|}{ Lesson planning } & \multicolumn{2}{c}{ Receptive skills } \\
\cline { 2 - 7 } & Palabra & IDL & \multicolumn{1}{|c}{ Palabra } & IDL & \multicolumn{1}{c}{ Palabra } & IDL \\
\hline 1 & Noun & 0,569931 & Subsidiary-aim & 0,725130 & Listening & 0,856188 \\
\hline 2 & Verb & 0,526206 & Anticipated-problem & 0,349800 & Reading & 0,810265 \\
\hline 3 & Adjective & 0,442522 & Main-aim & 0,344603 & Skimming & 0,337602 \\
\hline 4 & Subject & 0,351747 & Assessment & 0,288572 & Scanning & 0,301091 \\
\hline 5 & Pronoun & 0,294496 & AIDS & 0,250167 & Extensive-reading & 0,132490 \\
\hline 6 & Adverb & 0,269075 & Time & 0,221276 & Gist & 0,117202 \\
\hline 7 & Word & 0,157592 & Aim & 0,170204 & Listening-for-gist & 0,109087 \\
\hline 8 & Predicate & 0,153698 & Length & 0,169799 & Intensive-reading & 0,090888 \\
\hline 9 & Syntax & 0,143122 & Assumed-knowledge & 0,154537 & Reading-comprehension & 0,088240 \\
\hline 10 & Grammar & 0,141584 & Personal-aim & 0,138583 & Books & 0,074197 \\
\hline
\end{tabular}


- En el centro de interés assessment destaca el léxico relacionado con tipo de evaluación (formal assessmente informal assessment) y función de evaluación (summative test y formative test);

- En el centro de interés productive skills, los vocablos con el mayor IDL son writing y speaking. El resto de los ítems léxicos, en general, corresponden a tipos de producción textual (essay, letter, email, report y proposal) y oral (dialogue);

- En el centro de interés reflectivity se evidencia léxico relacionado con prácticas reflexivas de autocrítica (self-evaluation, self-assessment, feedback y self-reflection).

TABLA 8 - Léxico disponible en los centros de interés assessment, productive skills y reflectivity

\begin{tabular}{c|l|c|l|c|l|c}
\hline \multirow{2}{*}{} & \multicolumn{2}{|c|}{ Assessment } & \multicolumn{2}{c|}{ Productive skills } & \multicolumn{2}{c}{ Reflectivity } \\
\cline { 2 - 7 } & \multicolumn{1}{c|}{ Palabra } & IDL & \multicolumn{1}{c}{ Palabra } & IDL & Palabra & IDL \\
\hline 1 & Formal-assessment & 0,757461 & Writing & 0,907082 & Self-evaluation & 0,211575 \\
\hline 2 & Informal-assessment & 0,700233 & Speaking & 0,871016 & Self-assessment & 0,196785 \\
\hline 3 & Summative-test & 0,334941 & Essay & 0,246275 & Opinion & 0,174128 \\
\hline 4 & Test & 0,274970 & Letter & 0,160341 & Thinking & 0,163896 \\
\hline 5 & Formative-test & 0,273484 & Email & 0,117591 & Feedback & 0,151631 \\
\hline 6 & Feedback & 0,190423 & Report & 0,113048 & Improvement & 0,119607 \\
\hline 7 & Rubric & 0,176832 & Dialogue & 0,113032 & Thoughts & 0,113432 \\
\hline 8 & Diagnostic-test & 0,175113 & Proposal & 0,080348 & Experiences & 0,112378 \\
\hline 9 & Evaluation & 0,136897 & Production & 0,077791 & Personal & 0,104162 \\
\hline 10 & Monitoring & 0,127575 & Argumentative-essay & 0,075182 & Self-reflection & 0,103571 \\
\hline
\end{tabular}


- En el centro de interés methods and approaches in language teaching se observa la presencia de léxico relacionado con métodos (TBL, ${ }^{3}$ audiolingualism, silent way, grammar-translation and suggestopedia), modelos de enseñanza $\left(P P P^{4}\right.$ y $\left.E S A^{5}\right)$, técnicas $\left(T P R^{6}\right)$ y enfoques (communicative approach y behaviorism);

- En el centro de interés integrated language skills se presentan con el IDL más alto las cuatro habilidades lingüísticas básicas (speaking, listening, reading y writing), también se presentan vocablos en torno a la subdivisión de estas habilidades en productivas (productive skills) y receptivas (receptive skills), además de la aparición de algunas sub-habilidades (skimming y scanning).

TABLA 9 - Léxico disponible en los centros de interés methods and approaches e integrated language skills.

\begin{tabular}{c|l|c|c|c} 
& \multicolumn{2}{|c|}{ Methods and approaches } & \multicolumn{2}{c}{ Integrated language skills } \\
\hline & \multicolumn{1}{|c|}{ Palabra } & IDL & \multicolumn{1}{c}{ Palabra } & IDL \\
\hline 1 & TBL & 0,741784 & Speaking & 0,804644 \\
\hline 2 & PPP & 0,683378 & Listening & 0,743767 \\
\hline 3 & TPR & 0,590554 & Reading & 0,735351 \\
\hline 4 & Communicative-approach & 0,400082 & Writing & 0,728053 \\
\hline 5 & Audiolingualism & 0,270111 & Productive-skills & 0,147212 \\
\hline 6 & Silent-way & 0,254943 & Receptive-skills & 0,142882 \\
\hline 7 & Grammar-translation & 0,197644 & Approaches & 0,082383 \\
\hline 8 & ESA & 0,159685 & Skimming & 0,080798 \\
\hline 9 & Suggestopedia & 0,140146 & Scanning & 0,076637 \\
\hline 10 & Behaviorism & 0,133961 & Skills & 0,075850 \\
\hline
\end{tabular}

${ }^{3}$ TBL: Task based learning (aprendizaje basado en tareas).

${ }^{4}$ PPP: Presentation-practice-production (presentación-práctica-producción).

${ }^{5}$ ESA: Engage-study-activate (vincular-estudiar-activar).

${ }^{6}$ TPR: Total physical response (respuesta física total). 


\section{Conclusiones}

A partir de los resultados obtenidos se pueden extraer las siguientes conclusiones:

Con respecto a los centros de interés de tipo general, el NPD más alto se evidencia en los centros feelings and emotions y hobbies con un NPD de 215 y de 182, respectivamente. El XR más alto se presenta en food and drink y family members, con un XR de 25,39 y de 20,79, respectivamente. El último índice en estudio muestra que el IC más alto se manifiesta en los centros family members (IC $=0,29835)$ y body parts (IC $=0,22081)$.

Por otro lado, en los centros de interés de tipo especializado se observa que el NPD más alto se presenta en productive skills y lesson planning, con un NPD de 167 y de 162, respectivamente. El XR más alto se evidencia en lesson planning $(\mathrm{XR}=15,39)$ y language components $(\mathrm{XR}=12,46)$. Finalmente, los IC más altos se encuentran en los centros language components $(\mathrm{IC}=0,10052)$ y lesson planning $(\mathrm{IC}=0,09502)$.

En cuanto a la hipótesis planteada en el estudio; a saber, el promedio del léxico disponible de los estudiantes en los centros de interés de tipo general es mayor que el especializado. Los resultados muestran que el XR en los centros generales $(\mathrm{XR}=17,94625)$ es mayor que en los especializados $(\mathrm{XR}=10,95)$. Esto podría deberse al carácter restrictivo que tiene el léxico especializado desde la perspectiva cuantitativa (NAVARRO MARRERO, 2009), dado que corresponde a un léxico relacionado con un aspecto de formación disciplinaria que se utiliza al interior de una comunidad específica (profesores de inglés) y que en el contexto de la formación inicial docente se circunscribe, en general, a asignaturas del área de prácticas pedagógicas y didáctica del inglés. Al contrario, el léxico general corresponde a un léxico de uso cotidiano y frecuente, que puede aparecer en revistas, películas y canciones; y que en contextos de la formación inicial docente, sería transversal a todas las asignaturas que forman parte del programa de estudio.

Al comparar los centros generales con los especializados también se observa que los centros de tipo general presentan un índice de cohesión más alto que el de los centros especializados, IC $=0,14925$ y 0,07875875 , respectivamente. Esto indica que las respuestas en los centros de interés de tipo general tienen un mayor grado de coincidencia y que tenderían a ser léxicamente más cerrados y más compactos. Este fenómeno dependería de cuán restringido o semánticamente concreto es el tema del centro de interés 
(AYORA, 2006). Este es el caso de los centros generales family members, body parts y colors, en los cuales es posible encontrar un número limitado de vocablos que no permitiría una gran variación en las respuestas de los sujetos.

En relación con el tercer índice en estudio (NPD), los resultados no reflejan una diferencia significativa entre el NPD de los centros generales $(139,25)$ y el NPD de los centros especializados $(140,75)$. Esto significa que el número de palabras que conocen los sujetos de la muestra es similar en los dos tipos de centros. Sin embargo, el grado de coincidencia de los sujetos (IC) y el promedio de palabras (XR) son más altos en los centros de tipo general que en los especializados. Esto indicaría que los sujetos de la muestra comparten en una mayor medida el léxico de tipo general, lo que no ocurriría en el caso del léxico especializado. Este fenómeno podría explicarse debido a que los centros especializados son divergentes y tendrían un carácter más abierto y difuso, lo que permitiría una mayor variación en el léxico evocado por los estudiantes (AYORA, 2006).

Salcedo Lagos y Del Valle (2013) plantean que los resultados obtenidos a partir de una prueba de disponibilidad léxica configuran una muestra clara y precisa de las palabras que los sujetos conocen y están capacitados para utilizar en una situación comunicativa, lo que a la luz de los resultados evidencia el dominio léxico de los sujetos participantes en este estudio que les permitiría desenvolverse tanto en situaciones cotidianas como en contextos profesionales utilizando el idioma inglés como medio de comunicación. A partir de los resultados también es posible constatar que el léxico evocado por los estudiantes en los centros especializados es de tipo técnico, que es coherente con los cuatro años de formación en la especialidad que poseen los sujetos de la muestra (GÓMEZ PARRA; GUERRA SALAS, 2004; QUINTANILLA ESPINOZA; SALCEDO LAGOS, 2019).

No obstante, es necesario conocer el léxico que el estudiante debe adquirir durante su proceso de formación profesional para comunicarse con otros profesionales de forma más efectiva y/o si el léxico que ha sido adquirido es pertinente para desenvolverse el interior de dicha comunidad (MEDINA, 2009). Por esta razón, es relevante indagar en futuras investigaciones cuál es el léxico disponible de los académicos que forman a estos futuros profesores (reconociéndolos como un modelo a seguir), dado que esta exploración podría aportar información relevante para la toma de decisiones pedagógicas con respecto a posibles adecuaciones curriculares 
a nivel de entrenamiento lingüístico en el caso de los centros generales; y a nivel contenidos de la disciplina (HERRANZ LLÁCER, 2018) y de didáctica de aula en el caso de los centros especializados.

\section{Agradecimientos}

Los autores agradecen el financiamiento de Conicyt-CHA/Doctorado Nacional/2014- 21141227

\section{Contribución de los autores}

Angie Quintanilla Espinoza: propuesta de la investigación, recolección y tabulación de datos, desarrollo del marco teórico y bosquejo de análisis de resultados. Pedro Salcedo Lagos: profundización del diseño del artículo, análisis de resultados y conclusiones.

\section{Referencias}

AYORA, C. Disponibilidad léxica en Ceuta: aspectos sociolingüísticos. Cádiz: Universidad de Cádiz, 2006.

BONORINO, M.; CUÑARRO, M. Las relaciones léxicas en los procesos de lectura y escritura: diagnóstico y propuesta pedagógica. Filología, Buenos Aires, v. 38, p. 251-283, 2006.

CEPEDA, M.; GRANADA, M.; POMES, M. Disponibilidad léxica en estudiantes de primero básico. Literatura y Lingüistica, Santiago, n. 30, p. 166-181, 2014. Doi: https://doi.org/10.4067/S0716-58112014000200010

CHILE. Ministerio de Educación. Estándares orientadores para carreras de pedagogía en inglés. Santiago: Ministerio de Educación, 2014.

ECHEVERrÍA, M.; HERRERA, M.; MORENO, P.; PRADENAS, F. Disponibilidad léxica en Educación Media. Revista de Lingüística Teórica y Aplicada, Concepción, n. 25, p. 55-116, 1987.

FERNÁNDEZ, A. Gender and motivation in EFL vocabulary production. In: JIMÉNEZ CATALÁN, R. M. (ed.). Gender Perspectives on Vocabulary in Foreign Languages. Basingstoke: Palgrave Macmillan, 2010. p. 93-116. Doi: https://doi. org/10.1057/9780230274938_5

FERREIRA, A.; SALCEDO LAGOS, P.; DEL VALLE, M. Estudio de disponibilidad léxica en el ámbito de las matemáticas. Estudios Filológicos, Valdívia, n. 54, p. 69-84, 2014. Doi: https://doi.org/10.4067/S0071-17132014000200004 
FERREIRA, R.; ECHEVERRÍA, M. Redes semánticas en el léxico disponible de inglés L1 e inglés LE. Onomázein, Santiago, v. 21, n. 1, p. 133-153, 2010.

GARZÓN, A.; PENAGOS, L. Disponibilidad léxica en estudiantes de primer semestre de pregrado de una institución universitaria de Villavicencio, Colombia. Formay Función, Bogotá, v. 29, n. 2, p. 63-84, 2016. Doi: https://doi.org/10.15446/ fyf.v29n2.60189

GERMANY, P.; CARTES, N. Léxico disponible en inglés como segunda lengua en instrucción formalizada. Estudios Pedagógicos, Valdivia, n. 26, p. 39-50, 2000. Doi: https://doi.org/10.4067/S0718-07052000000100003

GÓMEZ PARRA, M. E.; GUERRA SALAS, L. Disponibilidad y fines específicos: análisis del centro de interés prensa. In: CONGRESO INTERNACIONAL DE LA ASOCIACIÓN EUROPEA DE LENGUAS PARA FINES ESPECÍFICOS, 3., 2004, Granada. Actas [...]. Granada: Universidad de Granada, 2004. p. 695-703. GOUGENHEIM, G. La statistique de vocabulaire et son application dans l'enseignement des langues. Revue de l'Enseignement Supérieur, Paris, n. 3, p. 137-144, 1967.

GOUGENHEIM, G.; MICHÉA, R.; RIVENC, P.; SAUVAGEOT, A. L'élaboration du français élémentaire: étude sur l'établissement d'un vocabulaire et d'une grammaire de base. Paris: Didier, 1956.

GUERRA SALAS, L.; GÓMEZ SÁNCHEZ, M. E. Español de los medios de comunicación: aspectos de disponibilidad léxica. In: CONGRESO INTERNACIONAL DE LA ENSEÑANZA DEL ESPAÑOL COMO LENGUA EXTRANJERA, 14., 2003, Burgos. Actas [...]. Burgos: Universidad de Burgos, 2003. p. 336-343.

HERNÁNDEZ, N.; TOMÉ, C. Léxico disponible en primera y segunda lengua: bases cognitivas. In: DE LA ROSA, F. D. B. (ed.). Palabras vocabulario léxico: la lexicología aplicada a la didáctica y a la diacronía. Venezia: Edizioni Ca' Foscari, 2017. p. 99-122.

HERRANZ LLÁCER, C. V. Disponibilidad léxica de los futuros profesores de Educación Infantil y Primaria. Revista Electrónica Interuniversitaria de Formación del Profesorado, Cuenca, v. 21, n. 1, p. 143-159, 2018. Doi: https://doi.org/10.6018/ reifop.21.1.295271

JIMÉNEZ, R.; LLACH, A.; FERNÁNDEZ, A.; CANGA, A. The effect of age on EFL learners' lexical availability: word responses to the cue words "town" and “countryside". In: JIMÉNEZ CATALÁN, R. M. (ed.). Lexical Availability in English and Spanish as a Second Language. Amsterdam: Springer, 2014. p. 37-51. 
JIMÉNEZ CATALÁN, R. M.; OJEDA ALBA, J. Disponibilidad léxica en inglés como lengua extranjera en dos tipos de instrucción. Lenguaje y Textos, Valéncia, n. 30, p. 166-176, 2010.

LEXMATH. Acerca del proyecto. Concepción: Lexmath, 2013. Disponible en: https://bit.ly/2ZKmw35. Acceso el: 25 jun. 2019.

LIGHTBOWN, P.; SPADA, N. How Languages Are Learned. 3. ed. Oxford: Oxford University Press, 2006.

LÓPEZ MORALES, H. Léxico disponible en Puerto Rico. Madrid: Arco Libros, 1999. Doi: https://doi.org/10.31819/9783865278852-011

LUGONES, A. El léxico disponible de los alumnos de secundaria bilingüe (español-inglés). 2015. Tesis (Doctorado en Lengua Española) - Universidad de Salamanca, Salamanca, 2015.

MATEO, M. Disponibilidad léxica en el COU almeriense: estudio de estratificación social. Almería: Universidad de Almería, 1998.

MEDINA, C. Disponibilidad léxica jurídica. 2009. Tesis (Magister en Lingüística) Universidad de Concepción, Concepción, 2009.

MENA, M. Disponibilidad léxica infantil en tres niveles de enseñanza básica. 1986. Tesis (Magister en Lingüística) - Universidad de Concepción, Concepción, 1986.

MICHÉA, R. Mots fréquents et mots disponibles: un aspect nouveau de la statistique du langage. Les Langues Modernes, [S. l.], n. 47, p. 338-344, 1953.

NAVARRO MARRERO, Y. Terminología especializada en el área de la fisioterapia: acercamiento desde la metodología de la disponibilidad léxica específica. Barcelona: Universidad Autónoma de Barcelona, 2009.

PACHECO, A.; PONCE CASTAÑEDA, S.; PALOMARES SÁNCHEZ, S. A. Disponibilidad Léxica Matemática en estudiantes de Ingeniería y Ciencias. Unión, [S.l.], n. 47, p. 44-61, 2016.

PALAPANIDI, K. La aplicación de la disponibilidad léxica a la didáctica del léxico de LE. Revista Nebrija de Lingüistica Aplicada, Madrid, v. 11, n. 6, p. 69-77, 2012.

PEDRONI TORRES, T. El léxico disponible de los profesores de ELE en formación en la ciudad de São Paulo. 2015. Tesis (Doctorado en Lingüística Aplicada) - Universidad de Salamanca, Salamanca, 2015.

QUINTANILLA ESPINOZA, A.; SALCEDO LAGOS, P. Estudio del léxico especializado en inglés como lengua extranjera en estudiantes de pregrado. Formación Universitaria, La Serena, v. 12, n. 4, 2019. 
SALCEDO LAGOS, P.; DEL VALLE, M. Disponibilidad léxica matemática en estudiantes de enseñanza media de Concepción, Chile. Atenas, Matanzas, v. 4, n. 21, p. 1-16, 2013.

SALCEDO LAGOS, P.; DEL VALLE, M.; QUINTANILLA ESPINOZA, A.; ZAMBRANO, C. Lexical availability of students in mathematics education in Southern Chile. In: ANNUAL INTERNATIONAL CONFERENCE OF EDUCATION, RESEARCH AND INNOVATION, 9., 2016, Seville. Actas [...]. Seville: ICERi, 2016. p. 3676-3683. Doi: https://doi.org/10.21125/iceri.2016.1870 ŠIFRAR KALAN, M. Lexical availability and L2 vocabulary acquisition. Journal of Foreign Language Teaching and Applied Linguistics, [S. l.], v. 2, n. 2, p. 191-200, 2015. Doi: https://doi.org/10.14706/JFLTAL152216

STOLLER, F. Content-based instruction. In: DEUSEN-SCHOLL, N. V.; HORNBERGER, N. H. (ed.). Encyclopedia of Language and Education: Second and Foreign Language Education. New York: Springer, 2007. v. 4, p. 59-70.

URZÚA, P.; SÁEZ, K.; ECHEVERRÍA, M. S. Disponibilidad léxica matemática: análisis cuantitativo y cualitativo. RL A, Concepción, v. 44, n. 2, p. 59-76, 2006. Doi: https://doi.org/10.4067/S0718-48832006000200005

VALENCIA, A.; ÁVILA, E.; MUÑOZ, G.; NÚÑEZ, N.; ECHEVERRÍA, M. S.; VÉLIZ, M. Evaluación de la riqueza léxica en estudiantes de último año de enseñanza media. Estudios Filológicos, Valdivia, v. 27, p. 59-72, 1992.

ZUBANOV, M. Disponibilidad léxica en alemán e inglés como lengua extranjera. 2006. Tesis (Magister en Lingüística) - Universidad de Concepción, Concepción, 2006. 


\section{ANEXO A: Test de Disponibilidad Léxica}

Datos del informante:

1. Sexo: Hombre Mujer

2. Edad:

\section{Instrucciones:}

Tendrás 2 minutos para escribir todas las palabras en inglés que se te vengan a la mente en cada una de las 16 temáticas que presentará el investigador.

No existe un mínimo ni un máximo de palabras esperadas, sólo debes escribir las palabras que se activen en tu memoria. Ejemplo:

Encuestador: Write all the words related to "Transport" you know.

\begin{tabular}{|l|}
\hline \multicolumn{1}{|c|}{ Transport } \\
\hline 1. Car \\
\hline 2. Bus \\
\hline 3. Plain \\
\hline 4. Truck \\
\hline (...) \\
\hline 25. Airplane \\
\hline
\end{tabular}

\begin{tabular}{|l|l|l|l|}
\hline & & & \\
\hline 1. & 1. & 1. & 1. \\
\hline 2. & 2. & 2. & 2. \\
\hline 3. & 3. & 3. & 3. \\
\hline 4. & 4. & 4. & 4. \\
\hline$(\ldots)$. & $(\ldots)$. & $(\ldots)$ & $(\ldots)$. \\
\hline 25. & 25. & 25. & 25. \\
\hline
\end{tabular}

Data de submissão: 01/02/2018. Data de aprovação: 07/03/2019. 